\title{
Chemical and Morphological Evolution of Nanoporous Pd/Rh Alloy Particles for Hydrogen Storage
}

\author{
J.D. Sugar*, B.W. Jacobs**, and D.B. Robinson*** \\ * Materials Physics Department, Sandia National Laboratories, Livermore, CA 94550 \\ ** Protochips, Inc., Raleigh, NC, 27606 \\ *** Energy Nanomaterials Department, Sandia National Laboratories, Livermore, CA 94550
}

$\mathrm{Pd}$ and Pd alloys have the ability to absorb large volumes of hydrogen isotopes [1]. By introducing a large surface area of pores into these alloys, it is possible to improve surface-limited reaction rates and the hydrogen storage properties. In addition, a continuous pore structure in Pd alloys can provide an escape path for the helium decay product when tritium is stored in them [2-4]. We synthesize nanoporous Pd and Pd alloys in a scalable fashion using surfactant templates $[5,6]$. The resulting particles are several hundred nanometers in diameter and have uniformly distributed $\sim 3 \mathrm{~nm}$ pores throughout the particles. Corresponding bright-field (BF) and high-angle annular dark-field (HAADF) STEM images of the as-fabricated particles are shown in Figure 1 a) and b). Alloying nanoporous $\mathrm{Pd}$ particles with a higher melting temperature metal such as $\mathrm{Rh}$ has been shown to increase the temperature range over which the nanoporous structure remains morphologically stable [5].

We will show the results of a suite of characterization techniques that we use to study the chemical and morphological stability of these nanoporous particles in different ambient environments. The results of multivariate statistical analysis on an EDS spectrum image are shown in Figure $1 \mathrm{c}$ ). The results show that after synthesis, the $\mathrm{Rh}$ is not uniformly distributed throughout the particles. Instead, the particles exhibit a core/shell structure with a Rh-rich shell. When these particles are subsequently annealed in a reducing environment, it is possible to locally redistribute the $\mathrm{Rh}$ more uniformly in the core of the particle. This is similar to what has been shown in nanoparticles using synchrotron radiation sources [7, 8]. The use of a low-drift in-situ heating holder allows us to collect morphological data as the particles are heated. The initial and final stages of a heating experiment to $870^{\circ} \mathrm{C}$ are shown in Figure 2 a) and b), respectively. The nanoporous structure has completely collapsed after the elevated temperature anneal. Movies recorded during the pore collapse process are drift corrected with a custom MATLAB script that keeps the same sample region in the field of view over a wide range of temperatures with little to no interference from the microscope operator. This allows us to study the kinetics and mechanisms of pore collapse in an individual particle. The relationships between the compositional and morphological evolution of these particles at elevated temperatures will be discussed.

\section{References}

[1] J.C. Barton, et al., Nature 197 (1963)

[2] G.J. Thomas, et al., Journal of Nuclear Materials 116 (1983)

[3] S. Thiebaut, et al., Journal of Nuclear Materials 277 (2000)

[4] D.F. Cowgill, Fusion Science and Technology 48 (2005) 539.

[5] D.B. Robinson, et al., International Journal of Hydrogen Energy 35 (2010)

[6] D.B. Robinson, et al., International Journal of Hydrogen Energy 34 (2009)

[7] F. Tao, et al., Science 322 (2008)

[8] F. Tao, et al., Journal of the American Chemical Society 132 (2010) 
[9] Sandia is a multiprogram laboratory operated by Sandia Corporation, a Lockheed-Martin Company, for the US-DOE-NNSA, under Contract DE-AC04-94AL85000.
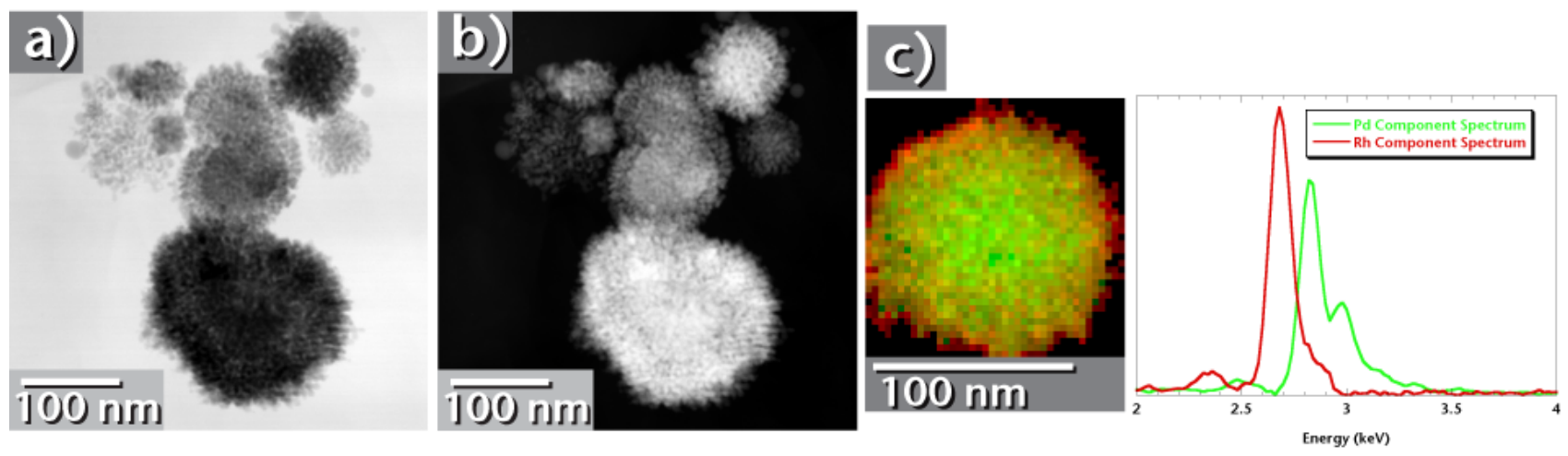

FIG. 1. BF-STEM a) and HAADF-STEM b) image of as-fabricated nanoporous particles. In c), the results of a multivariate statistical analysis are shows from an EDS spectrum image. The analysis can clearly resolve the $\mathrm{L}_{\alpha}$ x-ray lines for $\mathrm{Pd}$ and $\mathrm{Rh}$ and shows the core/shell microstructure of an individual particle. The core is $\mathrm{Pd}$ rich and the shell is $\mathrm{Rh}$ rich.
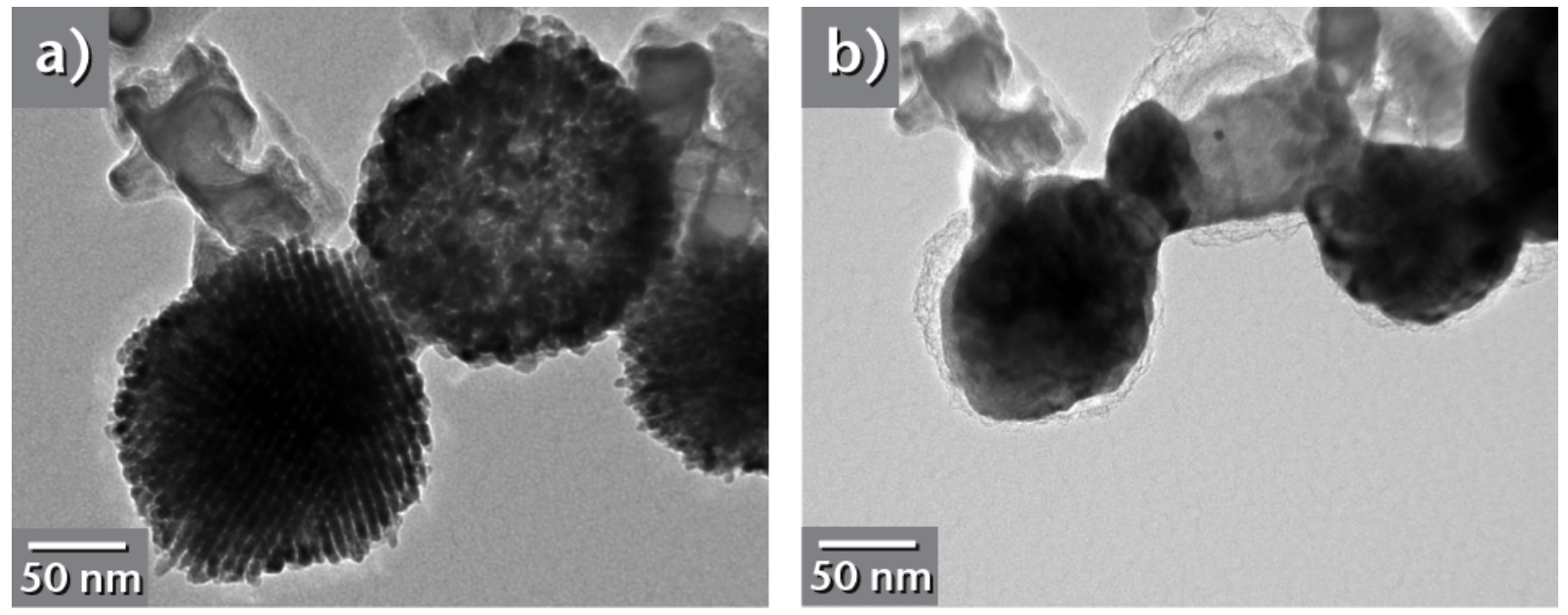

FIG. 2. BF images of nanoprous particles before, a), and after, b), an in-situ heating experiment to $870^{\circ} \mathrm{C}$. The collapse of the nanoporous microstructure is visible after heating the particles. 